\title{
La différence dans le système du même. Contribution philosophique à l'approche genre
}

\author{
Béatrice Faye*
}

\section{Résumé}

La réflexion sur la relation entre homme et femme a connu des moments importants dont chacun correspond à la prééminence d'un modèle théorique particulier. Partis d'un modèle « unidimensionnel » enraciné dans la dimension biologique, les chercheurs ont élaboré des modèles «pluridimensionnels » pour rendre compte de la complexité croissante de la catégorie du genre et de ses composantes. Dans l'histoire économique, la division genrée du travail est naturalisée à l'image de la différenciation biologique des sexes. De manière plus spécifique, on constate que les théories économiques restent généralement silencieuses sur la manière dont les rapports sociaux affectent le développement économique. Rien d'étonnant à cela puisque la science économique conventionnelle est mal armée pour répondre aux questions les plus fondamentales du développement. Elle n’est guère mieux équipée pour expliquer les rapports sociaux entre hommes et femmes. Elle constitue davantage un obstacle qu'un instrument pour l'analyse de ces rapports. En vérité, en mettant fin à la distinction des rôles, et en prenant pied systématiquement dans tous les domaines jadis réservés aux hommes, les femmes ont fait évanouir l'universelle caractéristique masculine : la supériorité de l'homme sur la femme. Avec la « conscience de genre », les femmes expriment leur sentiment d'appartenance à un groupe pensé non pas sur la base d'une distinction de classe par exemple, mais selon les catégories de sexes. Cette « conscience de genre " s'articule souvent à l’idée d'une supériorité des femmes comme gérantes de la famille, ferment de la société et remède aux maux de l’État.

* Sœur Béatrice Faye, Congrégation Immaculée Conception, Institution Notre Dame, Dakar, Sénégal. 


\begin{abstract}
Reflections on male-female relations have gone through important stages, each of which corresponds to a specific predominant theoretical model. Starting from a 'one-dimensional' model rooted in the biological dimension, researchers developed 'multi-dimensional' models to describe the increasing complexity of gender categories and its components. In the history of economics, gender-based division of labour is seen as natural, just as the biological discrimination between genders. More specifically, it is worth noting that economic theories generally remain silent about how social relations affect economic development. So, it is not surprising that conventional economics is neither well suited to address the most crucial issues of development, nor more relevant either to explain the male-female social relations. It is more of a hindrance than an instrument to analyse such relations. Indeed, by ending discrimination in roles while systematically participating in all the formerly male-reserved activities, women have swept away the universally-assumed characteristic of males: the superiority of men over women. Women could express a sense of belonging to a group defined, for example, not in terms of class distinction, but based on gender categories, although such 'gender consciousness' is often articulated around the idea of women's superiority as household managers, agents of social cohesion, and guardians against state excesses.
\end{abstract}

\title{
Introduction
}

La place accordée à la différence des sexes est un point « aveugle » de l'enseignement de la philosophie comme de l’histoire des idées en général. Le discours philosophique est le discours le plus fortement marqué par du masculin. En effet, on enseigne les « grands auteurs » ou les " grands systèmes », en négligeant leurs prises de position sur les questions relatives à ce sujet. Chez beaucoup de philosophes, un hiatus ininterrogé demeure entre la réflexion sur l’homme en général et la réflexion sur la répartition sexuée des places, des rôles, mais aussi des capacités cognitives, morales et esthétiques des femmes. Le pouvoir des hommes sur elles trouve son explication dans le rapport de force (la force physique) inscrit dans la nature, que la construction du droit vient soit organiser et réguler, soit amender sans l'abolir. C'est à croire que dès l'origine de l'humanité, leur privilège biologique a permis aux mâles de s'affirmer seuls comme sujets souverains. Pour Françoise Héritier en effet, « il y a peu de doute que la supériorité physique masculine et surtout l'alourdissement, l'immobilisation forcée et la fragilisation des femmes pendant la plus grande partie de leur vie en aient été les causes essentielles aux origines de l’humanité » (1978: 387). Autrement dit, le dualisme des sexes s’enracine dans la vérité du corps. Par la suite, l’idéologie s’est emparée de cette dichotomie première, et l'a étendue à tous les niveaux de la vie et à tous les aspects particuliers de la connaissance. 
C'est ainsi que le traitement de la question du rapport des femmes au savoir comporte deux volets principaux : le premier, d'ordre socio-historique, étudie les mécanismes par lesquels les femmes sont tenues à l'écart du savoir ou de certains domaines du savoir, ou n’y sont admises que comme instances d'exécution ou de diffusion mais non de création. Le deuxième volet concerne l'exercice même de ce savoir. Examiner le savoir scientifique à partir du paramètre de la sexuation en transforme-t-il l'appréhension ? On sait comment, dans le domaine des sciences humaines, cet abord de la question a pu être fécond, en raison du fait que ces sciences ont pour objet la réalité humaine dans son ensemble et que l'aborder en distinguant hommes et femmes et en analysant leurs rapports projette sur elle de nouveaux éclairages. " Il est décisif d'aborder une société, traditionnelle ou occidentale nous dit Collin, en s'interrogeant sur la place qu'y occupent les uns ou les autres, d'étudier de la même manière la structure de la famille, y compris dans sa forme pudiquement désignée comme « monoparentale », ou d'introduire ce paramètre dans les statistiques. Philosophie, Histoire, sociologie, économie même peuvent s'en trouver enrichies ou bouleversées » (Collin 1992 : 19).

Selon un schéma bien connu et inscrit dans la pensée psychanalytique, le savoir masculin aurait des affinités avec le détachable, la coupure, la séparation, le savoir féminin avec le global. Ainsi les hommes peuvent isoler un élément du réel, projeté hors d'eux, pour s'y consacrer dans l'oubli ou la négligence de ce à quoi il est rattaché ; les femmes quant à elles ne pensent jamais le détachement que tout au plus comme moment transitoire. « Dans une telle perspective, que l'on peut qualifier d'holiste, il n'y a pas de hiatus entre le savoir et la pensée, pas plus qu'il n'y en a entre la pensée et l'amour » (Collin 1992 : 21).

Concrètement, la non-visibilité ou l'occultation des femmes dans les actions qu'on appelle publiques montre à l'évidence le privilège de l'acteur masculin et sa centralité historique. De manière plus spécifique, on constate que les théories économiques restent généralement silencieuses sur la manière dont les rapports sociaux affectent le développement économique. Rien détonnant à cela puisque, la science économique conventionnelle est mal armée pour répondre aux questions les plus fondamentales du développement. Elle n'est guère mieux équipée pour expliquer les rapports sociaux entre hommes et femmes. Elle constitue davantage un obstacle qu'un instrument pour l'analyse de ces rapports.

Voilà pourquoi, la pensée féministe acculée à déconstruire et reconstruire sans cesse, utilise le genre comme outil d'analyse. Rappelons brièvement que le concept genre (gender en anglais) apparaît comme un des derniers des concepts herméneutiques introduits par le féminisme occidental. ${ }^{1}$ 
Il n’a pas été inventé par nous, nous en trouvons l'inspiration dans les études anthropologiques, sociologiques et culturelles de la littérature, anglo-saxonne essentiellement, consacrée aux rapports entre les rôles et les fonctions des hommes et des femmes dans nos sociétés. Cette médiation a été introduite particulièrement par les féministes américaines dans les années 80 et reprise un peu plus tard par les féministes françaises et québécoises. Ainsi, si nous partons de l'affirmation que le genre n'est pas simplement le fait biologique d'être un homme ou une femme, alors, on peut l'envisager comme, « une construction sociale, une façon d'être au monde, une façon d'être éduquée, une façon aussi d’être perçue, qui conditionne notre être et notre agir » (Mathieu 1991, 1992 ; Bissiliat 1992 ; CDEACF et Relais Femmes 1997). En d'autres termes, le concept de genre, au-delà des réalités biologiques des deux sexes, englobe la manière propre d'être et d'agir dans le monde, d'établir des relations, donc de marquer la différence, d'en revendiquer le droit, d'en souligner les potentialités, d'orienter une politique.

La médiation du genre comme instrument, outil, permet de mieux comprendre la place de la femme dans l'économique. Sans absolutiser cette médiation, elle constitue un outil important pour comprendre, par un biais différent, la complexité des relations humaines. C'est donc un outil en vue de la transformation des relations sociales, soit au niveau public, soit au niveau domestique de notre existence. Son intérêt se trouve dans sa capacité à offrir une nouvelle clé de lecture des réalités sociales et dans sa pertinence pour saisir toute forme de situation d’inégalités qu'elle soit liée au sexe, à l'ethnie ou à l'âge.

L'approche genre permet de cerner la position particulière des femmes dans l'économie et de faire le lien entre les dynamiques sociales. Ce qui nous amène à comprendre « quelque chose » de plus sur le « destin » de la femme. Le concept de genre nous révèle que non seulement le masculin est normatif dans les langues et la science, mais que la compréhension même de l'économie et du commerce ainsi que les formes proposées pour les apprécier, sont accordées à des modèles masculins. Il s'agit ici d'une espèce de « globalisation » du savoir et du pouvoir présente dans le monde patriarcal et dont il faut dénoncer les effets néfastes dans l'histoire des femmes et certainement de toute l'humanité. Si nous sommes contre les discours totalitaires, nous ne pouvons pas ériger un concept en clef explicative de la totalité. Il faut donc toujours garder la dialectique des rapports hommes/femmes, étant donné que la vie humaine se déroule dans l'interaction continuelle entre les deux sexes.

Reconnaissons-le, le genre est un concept abstrait. Il est parfois source de confusion, car il ne désigne pas uniquement la femme ; il fait aussi référence à l'homme. Le fait d'utiliser les deux termes de manière interchangeable est une erreur conceptuelle. Le terme genre s'utilise pour cerner les rôles 
sexuels. Il définit les valeurs et attitudes qu'une communauté ou une société juge appropriées à l'un ou l'autre sexe. Il résulte donc du processus de socialisation, assignant aux hommes et aux femmes des rôles différents au niveau de la production et de la distribution des responsabilités. Malgré les similitudes entre les différents courants féministes, il faut préciser qu'il n’y a jamais eu d'accord unanime sur la compréhension du rôle de la différence et sur le pouvoir de la différence. En effet, les discussions axées sur l'opposition entre hommes et femmes n'ont pas permis d'avancer beaucoup au niveau théorique. En revanche, la différence de genre est une différence parmi la multiplicité des différences. Il y a des différences entre hommes et femmes, entre hommes et hommes, entre femmes et femmes. Ces différences sont croisées par les différences d'âge, de culture, de religion et par bien d'autres.

Même s'il reste admis que la fuite en avant vers la globalisation où nous précipite la pensée néolibérale « unique » est d'expression essentiellement masculine ; le concept de genre, plus encore lorsqu'il est croisé à l'économie, est dans son essence un concept critique de l'organisation de la société. Il oblige donc à des remises en question à tous les niveaux, personnels et globaux. L'avènement de la globalisation constitue une opportunité dans notre approche genre. "Aujourd'hui plus que jamais nous dit dans ce sens Yvonne Gebara, les défis qui nous invitent à repenser l'épistémologie nous sont posés par le métissage croissant des cultures, la globalisation même des cultures, imposée par la mondialisation de l'économie, par les moyens de communication, et, très particulièrement, par l'éveil de la conscience des femmes qui cherchent à être reconnues comme sujets historiques à part entière » (Gebara 1999 : 107). Quand on introduit la médiation du genre en économie, une manière différente de comprendre les rapports sociaux s'impose. Penser l'être humain autrement semble être un défi absolument nécessaire de nos jours. Il nous faut construire des relations plus justes et une grande solidarité dans le monde en vue d'une mondialisation éthiquement durable. C'est à partir de cette perspective qui nous dépayse, qui nous invite à changer nos points de repère traditionnels, que nous entamons ce long processus de questionnement.

Notre premier axe de réflexion portera sur l'évolution conceptuelle des approches sur la femme afin de mettre en évidence la "cécité » des conceptualisations philosophiques, sociologiques et économiques à son égard. Ce qui nous permettra de les requestionner et les interroger à travers l'approche genre.

Le genre en questions dans les théories économistes constituera le second axe de notre travail. QUESTIONS avec (S) est à entendre ici d'un double point de vue. D'abord comme une remise en question de l'économie, ensuite, l'approche genre ${ }^{2}$ comme outil d'analyse et de réflexion part de 
cette interrogation pour repenser ce qui peut constituer pour l'humanité de nouvelles raisons de vivre, de croire et d’espérer. D’où notre approche philosophique de la question.

Nous avons tenté de limiter le champ très large et compliqué de « globalisation, genre et commerce » aux questions suivantes : pourquoi une personne veut ce qu'elle veut, et comment une personne rationnelle, qui s’occupe de son intérêt personnel, cherche ce qu'elle veut ? Quelle est la dimension sexuée de la définition traditionnelle des concepts tels que le bienêtre, le choix, la richesse et l'échange ? Nous tenterons par là d'entendre, et de faire entendre, la complexité, voire le désordre, inhérents à tout changement et encore à tout phénomène de mutation accélérée amplifié par un libéralisme débridé et une mondialisation implacable.

\section{Évolution conceptuelle des approches sur le genre}

\section{Une longue tradition d'inégalité : la métaphysique des sexes}

La métaphysique des sexes, qu'on peut aussi qualifier d'essentialisme, affirme une différence essentielle, voir naturelle, entre les femmes et les hommes, et définit leurs spécificités respectives. Comment la question des femmes et de la différence des sexes prend-elle place et se déplace-t-elle dans les dispositifs philosophiques jusqu'à nos jours ? L'exclusion remonte aux origines de la philosophie avec, Anaxagore, Socrate, Platon Aristote et Thomas d'Aquin. De plus, par sa nature, la femme se voit interdite de progrès. C'est ce que tentent de nous démontrer quelques philosophes choisis pour leur clarté sur la question. Il s’agit de : Pufendorf, Locke et Kant. A défaut de pouvoir éliminer complètement le principe féminin, tous s’emploient à le dévaloriser d'une manière ou d'une autre.

\section{L'égalité des femmes liée au devenir des hommes}

Pour Anaxagore, la détermination du sexe vient du père. Les concepts à l'aide desquels Platon et Aristote représentent le monde reflétaient déjà avec la même clarté « les lois de la physique, l’égalité des citoyens, et l'infériorité des femmes, des enfants et des esclaves » ; leur prétention à la validité universelle exprimait déjà le rapport de forces existant sur la place de l'agora.

Dans le Ménon, nous voyons comment Socrate ironise sur le propos de son interlocuteur selon lequel il y aurait des vertus propres à chaque sexe car les vertus viennent de l'âme et l'âme n'a pas de sexe. En revanche, dans le Timée est suggérée une certaine infériorité de la raison féminine par rapport à la raison masculine. Ainsi, ceux qui cherchent la procréation par le corps se tournent vers les femmes, et ceux qui cherchent la sagesse préfèrent la procréation par l'esprit, et se tournent vers les hommes. On voit ainsi réapparaître en divers points la doctrine platonicienne des affirmations selon lesquelles 
les femmes auraient des tendances les portant aux activités inférieures ou auraient les mêmes qualités que les hommes mais moins affirmées. On peut penser qu'il s'agit de dérives dues à la nature : la loi est faite pour les endiguer et révéler une capacité féminine fondamentalement égale à celle des hommes.

En retraversant les différents dialogues, on peut voir que l'affirmation égalitaire qui se fonde sur un présupposé d'identité minimisant tout ce qui pourrait le contester est souvent mise à l'épreuve. L'égalité est suspendue à l'identité, une identité calquée sur le modèle masculin, qui rejette dans l'ombre les caractéristiques féminines mais non celles des hommes prises comme référence. Dans le Timée, Platon soutient que les hommes qui, dans leur première vie, « étaient lâches ou qui passaient leur vie dans l'injustice furent changés en femmes à la seconde naissance »(Platon 1961 : II, 52), attestant ainsi de ce qu'être femme est une punition du sort. L'égalité des femmes semble liée à leur devenir hommes. Tout en posant le principe de l'égalité des hommes et des femmes, Platon le ruine par l'affirmation que «toutefois la femme est en toute chose inférieure à l'homme » (Platon 1984 : VII, 454)

Dans la République, où la partition du privé et du public est supprimée, les enfants étant élevés en commun, les femmes sont appelées aux mêmes charges publiques que les hommes. [... « Ce qu'on a donné aux hommes, c'est la musique et la gymnastique ; aux femmes aussi, il faudra donc que soit attribuée cette double discipline, avec ce qui regarde la guerre, et il faudra qu'elles soient employées aussi dans les mêmes conditions...]» ( Platon 1984 : I, 1021-1028). Ainsi, les mêmes capacités de l'âme sont présentes chez les hommes et les femmes. Les uns et les autres méritent la même éducation. Mais à aucun moment n'est envisagée la décision des femmes elles-mêmes, pas plus que n'est remise en cause leur subordination générale aux hommes.

Sur le thème de la génération et de la détermination du sexe, Aristote a élaboré un des plus beaux modèles explicatifs qui soient, modèle philosophique, complet, argumenté et raisonné. La question posée est la suivante : qui détient la supériorité ? La terre qui reçoit la semence ou la semence qui fertilise la terre ? Plusieurs réponses ont été données dont celle d'Aristote purement génétique que nous allons examiner.

L'écart de type génétique : l'hypothèse d'Aristote et de Thomas d'Aquin. Deux interrogations majeures se dégagent de la pensée d'Aristote : si la distinction forme-matière caractérise non seulement les êtres humains mais aussi l'ensemble des êtres du cosmos, comment peut-elle être ramenée à la distinction des sexes ? De plus, si tous les êtres humains partagent la même forme et sont semblables du point de vue métaphysique, comment penser les « femelles » comme l'écart monstrueux de l'humain parfait? 
Dans un long développement, Aristote, contrairement à ses prédécesseurs, envisage cette différence fondamentale en qualité de chaud et froid, qui implique et justifie la différence anatomique des organes.

Les uns dit-il prétendent que cette opposition existe dès le début, dans les germes, par exemple, Anaxagore et d'autres naturalistes disent que le sperme vient du mâle, que la femelle fournit le lieu. Le mâle vient de droite, la femelle de gauche, comme dans l'utérus les mâles sont à droite et les femelles à gauche. Pour d'autres, comme Empédocle, la différenciation se fait dans la matrice. D’après lui, les germes qui pénètrent dans un utérus chaud deviennent mâles, et femelles dans un utérus froid, et la cause de cette chaleur et de ce froid, il l'attribue au flux menstruel, suivant qu'il est plus froid ou plus chaud, plus ancien ou plus récent. Quant à Démocrite d'Abdère, il affirme bien que la différenciation de la femelle et du mâle a lieu dans la mère, mais d'après lui ce n'est pas la chaleur ou le froid qui font dans un cas une femelle, dans l'autre un mâle, c'est la prédominance du sperme de l'un des parents, ce sperme venant de la partie par laquelle se caractérisent la femelle et le mâle (Aristote 1963 : 136-149).

Le mouvement, c'est ce qui vient de l'homme et caractérise sa puissance générique et individuelle. La matière est ce qui vient de la féminité. Par principe, il faut entendre le premier moteur, que l'action soit menée en lui-même ou en un autre être. Pour Aristote donc, la « faiblesse congénitale » de la femme « atteint l’âme elle-même » et elle n’a ni la faculté de « délibérer » ni celle de « décider » (Aristote 1963 : I 1260a). L’homme et la femme sont de même inégaux dans la procréation : c’est le mâle qui transmet l'humanité, porteuse du principe divin ; la femme n'apporte que la matière (Aristote 1963 II, 1, 412a). Le principe actif de la vie est la semence masculine, la femme n’est qu'un réceptacle passif.

À part le fait que le principe de la matière introduit la corruption ${ }^{3}$ et la mort dans l'univers, il est cause aussi de la monstruosité. C'est dire très nettement la responsabilité maternelle du monstre. La monstruosité proprement dite s'applique au cas où l'engendré n'est pas de même espèce que le géniteur. Une simple dissemblance suffit à constituer une monstruosité au sens large : c'est ainsi que la femelle engendrée au lieu d'un mâle est un monstre. "Le tout premier écart du type génétique est la naissance d'une femelle au lieu d'un mâle » (Aristote 1963 livre IV, 2). Aristote a beau affirmer que ce monstre qu'est la femelle est nécessaire pour sauvegarder la différence de sexes, la femme n’en est pas moins présentée comme un échec de l'humanité. « Les femelles sont par nature plus faibles voire plus froides, et il faut considérer leur nature comme une défectuosité naturelle. Il y aura monstruosité également lorsqu'un enfant mâle ressemble à sa mère » (Aristote 1963 livre IV, 6, 775a). 
Plus tard, Thomas d'Aquin empruntera à Aristote l'idée que l'homme et la femme s'opposent de la même façon que la forme et la matière. Sur la différence des sexes, il est prolixe. Son originalité tient à son effort pour adapter les enseignements d'Aristote et les rendre compatibles avec la tradition patristique. Sa réhabilitation de la Nature et de la Raison confère à sa théologie un caractère particulièrement ouvert et « libéral ». C’est précisément parce qu'il s'efforce de réhabiliter la raison humaine que le fait d'en priver la femme a des conséquences plus graves. Thomas d'Aquin distingue en effet la sujétion de l'esclave et celle de la femme : la première n'est qu'une conséquence du péché alors que si la seconde est naturelle c'est qu’il aurait manqué à la multitude humaine ce bien qu'est l'ordre, si les uns n'avaient pas été gouvernés par d'autres plus sages. C'est par ce genre de sujétion précisément que, par nature, la femme est soumise à l'homme, car par nature, l'homme est plus largement pourvu du discernement de la raison. Tout cela ne manque pas de s'inscrire dans une vision politique et sociale que la postérité ne fera que développer.

\section{Non-visibilité et sur-visualisation dans l'institution sociale de Hobbes à Rousseau}

Forgée au XVIIe siècle par les théoriciens du droit naturel, la notion de pacte social est largement répandue au XVIIIe siècle. La théorie du contrat social, sous sa forme classique du pacte de soumission, fait alors autorité. Elle fait pratiquement partie du sens commun. Locke et Rousseau lui donneront un sens nouveau. Mais nous savons que cette notion avait déjà un sens très différent chez Hobbes et chez Pufendorf. En un certain sens, on pourrait étudier tous les philosophes politiques des XVIIe et XVIIIe siècles à partir de leur théorie du pacte social. Mais ce n'est pas en tant que tel l'objet de notre travail.

En revanche, il nous semble important de montrer comment ces différentes conceptions du contrat social reposent en dernière analyse sur des visions divergentes de la femme. On peut ainsi comprendre Hobbes, Pufendorf, Locke et Rousseau d'une manière unifiée. Leur démarche est la même : ils se posent la question de la place de la femme dans le pacte social.

\section{Égalité dans l'état de nature, domination dans l'état politique}

Hobbes estime qu'à l'état de nature, les femmes sont égales aux hommes car il n'existe pas, entre les deux sexes, une différence de force ou de prévoyance qui puisse déterminer le droit sans guerre. Sans doute, Hobbes se soucie-t-il peu de la liberté des femmes. L'enjeu de son affirmation subversive est autre : il veut établir que la domination, toute domination, est d'origine politique, contractuelle, volontaire, issue du consentement. Il envisage 
donc l'égalité dans l'état de nature et la domination dans l'état politique. C'est ce qui nous amène à envisager la femme dans cette sphère.

Le point de départ de l'œuvre majeure de Hobbes publiée en 1651, le Léviathan (Hobbes 1999) est que l'homme, individualité corporelle, est mu par la puissance de ses désirs. La démarche par laquelle il inscrit dans l'état naturel de l'homme l'obligation de " choisir » l'assujettissement se veut avant tout logique et scientifique. À l'état de nature, les animaux humains sont des machines mues par le mouvement vers l'assouvissement de leurs désirs sans autre rapport que celui qui les oppose entre eux dans leur désir d'acquérir une même chose. D’où la nécessité d'un acte volontaire par lequel ces individus/loups, qui sont toutefois dotés de raison, se fabriquent une seconde nature, une volonté unique, en aliénant définitivement leur liberté, leur droit naturel, au profit de la toute-puissance du monstre Léviathan, machine et « homme artificiel » à la fois, qui, « par la terreur qu'il inspire », les unifie et les protège tous (malgré eux) contre les ravages de cet autre monstre biblique qu'est le Béhémoth de la guerre civile.

Conformément au savoir scientifique de son époque, Hobbes ne nie pas que l'homme soit «l'agent principal de la génération ». Mais il affirme que Dieu lui a donné en la femme un " auxiliaire », qui possède naturellement la même capacité de tuer. Et qui, en outre, possède le privilège exclusif de savoir qui est le père de l'enfant. Si le pouvoir venait de la génération, ce serait à la mère que reviendrait naturellement le pouvoir.

D'où il suit, que par le même droit, un enfant est sous la domination immédiate de celui qui le premier le tient en sa puissance. Or, l'enfant qui vient de naître est en la puissance de sa mère, avant de se trouver en celle d'un autre, de sorte qu'elle peut l'élever ou l'exposer, ainsi que bon lui semble et sans qu'elle en soit responsable envers personne (Hobbes 1996 : 157).

Hobbes paraît suivre ici la logique : le père ne peut avoir de pouvoir sur l'enfant que si la mère consent à le lui céder. Ce n'est pas la génération, mais le consentement qui fonde donc la domination. Aussi bien semble-t-il admettre que la famille, tout comme l'État et toute autre association humaine, vient d'un contrat, d'un acte consenti qui soumet volontairement les individus à un pouvoir un et indivisible comme le souligne ce large extrait :

La domination s'acquiert de deux façons : en engendrant et en subjuguant. Le droit de domination issu de la génération est celui que le parent a sur ses enfants ; on parle alors de domination paternelle. Ce droit ne dérive pas de la génération, en ce sens qu'il appartiendrait au parent de dominer son enfant du seul fait qu'il l'a procréé ; il dérive du consentement de l'enfant, explicite ou manifesté par des preuves suffisantes. En effet pour ce qui est de la génération, Dieu ayant attribué à l'homme un auxiliaire, il y a toujours deux parents, qui le sont également l'un et l'autre : la domination de l'enfant devrait donc 
appartenir également aux deux, ce qui est impossible, car nul ne peut être soumis à deux maîtres. Sans doute, certains ont attribué la domination au seul père, alléguant la supériorité du sexe masculin : mais c'est là un faux raisonnement. En effet, il n'existe pas toujours, entre l'homme et la femme, une différence de force ou de prévoyance telle que le droit puisse être déterminé sans guerre. Ce litige est tranché par la loi civile ; et dans la plupart des cas (pas toujours cependant) la sentence est favorable au père, parce que dans la plupart des cas les Républiques ont été fondées par les pères, et non par les mères de famille » (Hobbes 1999 : 208-209).

En révélant aussi radicalement le caractère artificiel et conventionnel de toute domination politique, Hobbes, rompt avec les métaphysiques naturalistes antérieures. Mais, paradoxalement, en prétendant ainsi mieux fonder l'absolutisme, il le fragilise. Qui dit convention dit instabilité : ce que l'homme fait, il peut le défaire. Concernant les femmes, Hobbes ne parvient pas à assumer jusqu'au bout sa rupture avec le naturalisme. Abandonnant ici l'hypothèse logique pour lui substituer une hypothèse historique, il réinscrit dans la nature des choses

une institution manifestement incompatible avec l'hypothèse d'une nature des hommes/champignons. Il fait donc peser un doute durable sur la validité de sa construction de la nature humaine et de l'artifice politique. Si les familles étaient, comme il l'affirme, des "petits royaumes », existant déjà à l'état de nature, il y aurait des individus qui seraient naturellement soumis à des " petits souverains ». Et si ce sont ces petits souverains qui ont créé l'artifice de la République, celle-ci n'est donc pas le produit volontaire d'individus libres et égaux, mais se compose déjà de groupes organiques pré-politiques.

Par conséquent, si pour les femmes, la démonstration du caractère artificiel et conventionnel du pouvoir laisse la place aux coutumes, à l'état de fait, à l'ordre naturel des choses, c'est qu'elles n'ont pas volontairement participé à la création de l'État. L'argument se retourne contre Hobbes : car si les femmes ne sont pas, à l'égal des hommes, les véritables auteurs du Léviathan, pourquoi auraient-elles l'obligation de lui obéir ? Pufendorf et J. Locke nous donnent quelques éclaircissements sur la question à travers le pacte de sujétion.

\section{Le pacte de sujétion : du droit politique au droit domestique.}

À l'origine de la formation de l'État, Pufendorf voit un double contrat : d'union puis de soumission. Pour leur défense mutuelle, les hommes s'unissent par une première convention. Mais ce pacte d'union ne garantit pas la paix. L'homme, en effet, n'a pas une inclination naturelle suffisamment forte pour qu’elle le conduise spontanément à « vouloir » la société politique. Aussi, faut-il supposer qu'une seconde convention vienne compléter la première, une convention de soumission, un pacte de sujétion. En insistant sur la « ré- 
ciprocité du contrat, Pufendorf s'efforce aussi d'établir la nature « morale » des rapports juridiques et politiques. A la suite de quoi, le contrat s'infiltre partout, envahissant non seulement le droit politique et international, mais aussi le droit domestique. D’où l'équivoque de la notion de contrat de soumission qui tend à légitimer le commandement et les privilèges. De plus, ce deuxième contrat tient son caractère obligatoire du fait de la volonté divine. Ainsi sacralisé, il déploie toutes ses conséquences absolutistes et interdit notamment d'envisager un quelconque droit de résistance.

Voilà pourquoi, dans le statut que Pufendorf confère à la femme, on trouve la trace de cette ambiguïté, voire de l'incohérence de ces propositions. Certes, il remet en cause les fondements divins ou naturels de l'autorité maritale et insiste sur le caractère contractuel du mariage. Le pouvoir de l'homme sur l'homme, étant une réalité morale, ne peut exister sans un acte humain. Or aucune femme n'est obligée d'obéir à son mari avant de s'être soumise par son propre consentement à la volonté de son mari. Au fond, Pufendorf procède comme pour l'État. Il fait subtilement avaliser par le consentement une réalité d'ordre naturel à laquelle il confère un fondement juridique propre. Il ne prétend pas qu'existe une « nature » féminine, distincte de celle de l'homme, mais fait intervenir d'autres considérations que celles de droits des individus : la finalité du mariage, le bon ordre de la société, les traditions patriarcales, selon lesquelles c'est la femme qui entre dans la famille du mari, non l'inverse. Bref, au nom de ces considérations, la femme ne peut que vouloir la domination de l'homme.

Fondée sur la sujétion naturelle des femmes, la distinction privé-public introduite par Locke dessine comme nous le verrons le périmètre dans lequel sera contenue la liberté des femmes en tant que sujets politiques.

Supériorité générale de tout homme sur toute femme.

Lieu de liberté privée pour le propriétaire-chef de famille, l'espace domestique devient pour la femme lieu de privation de cette liberté première qu'est la propriété dont Locke fait « l'origine et la fin de toute République ». Il « prive » ainsi les femmes de la propriété de leur corps et de leur force de travail dont les produits reviennent à la gestion du mari. Il les prive aussi du droit de « léguer leur fortune à ceux qui leur sont agréables », et de l'autorité que confère ce pouvoir de transmission des biens. Locke n'a certes pas thématisé, comme l'a fait Machiavel l'exclusion des femmes du politique. Mais en recourant à la nature pour fonder la puissance conjugale, il affirme la supériorité générale de tout homme sur toute femme, dans et en dehors de la famille. Sa distinction privé-public dépouille les femmes non seulement de l'autonomie requise pour une participation active dans la vie politique, mais aussi des « raisons » pour lesquelles les hommes ont consenti à l'institution de la so- 
ciété politique. N’appartenant ni à la catégorie de propriétaires autonomes ni à celle de travailleurs qui vendent « librement » leur force de travail, elles n’ont ni la liberté requise ni les « raisons » de signer ce contrat qui fonde l’obéissance de la majorité de la population masculine aux lois civiles. Naturellement inférieures et pourtant autorisées à négocier, les femmes deviennent, du fait de « l'inconsistance » de cette théorie lockienne, une anomalie dans la communauté politique moderne. Impliquant la supériorité de « tous » les hommes, le fondement dont Locke dote la puissance conjugale implique la sujétion de «toutes » les femmes, y compris celles qui ne sont pas mariées. Dans ce cas, à qui, les célibataires et les veuves doivent-elles obéissance ? Sans doute pas à tous les hommes, à moins que tous les hommes ne s’accordent à leur donner les mêmes ordres. Au regard de son immense influence, on mesure les conséquences ravageuses de sa misogynie.

En tout ce qui n'est pas sexe, la femme est homme

(Rousseau $1966 \mathrm{~V}$ : 465)

Dans ce raccourci tient toute l'ambiguïté de Rousseau. Depuis des siècles, son œuvre fait l'objet d'interprétations aussi passionnelles que contradictoires. Il n’y a pas lieu, ici, de discuter de cette diversité de lectures. Retenons seulement que, à l'origine des questionnements les plus inquiets, on trouve le statut complexe et déroutant que Rousseau assure à la nature. Scrutant l'origine de l'inégalité parmi les hommes, il déclare superbement « écarter tous les faits ». L'homme est libre comme l'animal, à cette nuance près que la nature programme l'animal en toutes ses actions alors que l'homme y concourt « en qualité d'agent libre ».

Bref, aucune nécessité extérieure ne saurait peser sur les choix et les volontés d'un homme dont la sensibilité se perfectionne par l'exercice de la raison : ce n'est donc qu'un « concours fortuit de causes étrangères » qui va le rendre méchant en même temps que civilisé. Une suite d’accidents et de contingences engendre corruption, concurrence et inégalité. Confrontés à un tel malheur, les hommes choisissent d'organiser leur sécurité et de protéger leurs propriétés.

Puisque la Nature parle plus clairement en ce qui concerne les femmes, il serait absurde selon Rousseau de ne pas la laisser parler et de la contredire en éduquant les filles comme des garçons. Du point de vue de son sexe, la femme est femme mais, pour le reste, elle appartient, comme l'homme à l'espèce humaine. En d'autres termes, la femme est davantage une « vraie » femme : « le mâle n'est mâle qu'en certains instants, la femelle est femelle toute sa vie ou du moins toute sa jeunesse »; tout la rappelle sans cesse à son sexe, et pour en bien remplir les fonctions, il lui faut une constitution qui s’y rapporte » (Rousseau $1966 \mathrm{~V}: 470$ ). 
En somme, l'enfermement des femmes dans la famille rousseauiste fait partie d'un effort pour réconcilier l'individualisme du droit naturel avec les valeurs de la communauté, la liberté et l'autonomie individuelles avec la solidarité et la réciprocité indispensables dans la vie en société. C’est pourquoi, « la femme a plus d'esprit, et l’homme plus de génie ; la femme observe, et l'homme raisonne : de ce concours résultent la lumière la plus claire et la science la plus complète que puisse acquérir de lui-même l'esprit humain, la plus sûre connaissance, en un mot, de soi et des autres qui soit à la portée de notre espèce. Et voilà comment l'art peut tendre incessamment à perfectionner l'instrument donné par la nature » (Rousseau $1966 \mathrm{~V}$ : 466).

Ainsi, lorsque Rousseau s'attache à définir le couple idéal, Émile et Sophie, c’est volontairement qu'il « fait » de celle-ci le complément de celui-là. Ayant longuement défini Émile comme une créature active, impétueuse, forte, courageuse et intelligente, le philosophe trace le portrait d'une épouse passive, timide, faible et soumise. « Faite spécialement pour plaire à l’homme ", Sophie sera élevée pour être coquette, peu intelligente et se contenter des seconds rôles... Telle est sa "nature” de n’avoir pas été créée pour ellemême, mais «pour être subjuguée par l’homme... lui être agréable... lui céder et supporter même son injustice » (Rousseau 1966 V : 693-731). Autrement dit, la femme et l'homme sont faits l'un pour l'autre, mais leur mutuelle dépendance n’est pas égale : « les hommes dépendent des femmes par leurs désirs ; les femmes dépendent des hommes et par leurs désirs et par leurs besoins ; nous subsisterions plutôt sans elles qu'elles sans nous. Pour qu'elles aient le nécessaire, pour qu'elles soient dans leur état, il faut que nous le leur donnions, que nous voulions le leur donner, que nous les en estimions dignes ; elles dépendent de nos sentiments, du prix que nous mettons à leur mérite, du cas que nous faisons de leurs charmes et de leurs vertus » (Rousseau 1966 V : 731). Ainsi, pour la préparer à sa « vocation » d'épouse et de mère, il est nécessaire de lui former un caractère doux, l'exercer à la contrainte, lui apprendre que la « dépendance est un état naturel aux femmes » (Rousseau $1966 \mathrm{~V}$ : 731).

À l'aide d'un tableau, nous avons condensé les opinions toutes faites des hommes et des femmes sur eux-mêmes. En observant bien ces stéréotypes (Rocheblave-Spenle 1964 : 164), on est frappé par les éternelles oppositions marquées du signe plus ou du signe moins. 


\section{Tableau 1 : Trait du stéréotype par sexe}

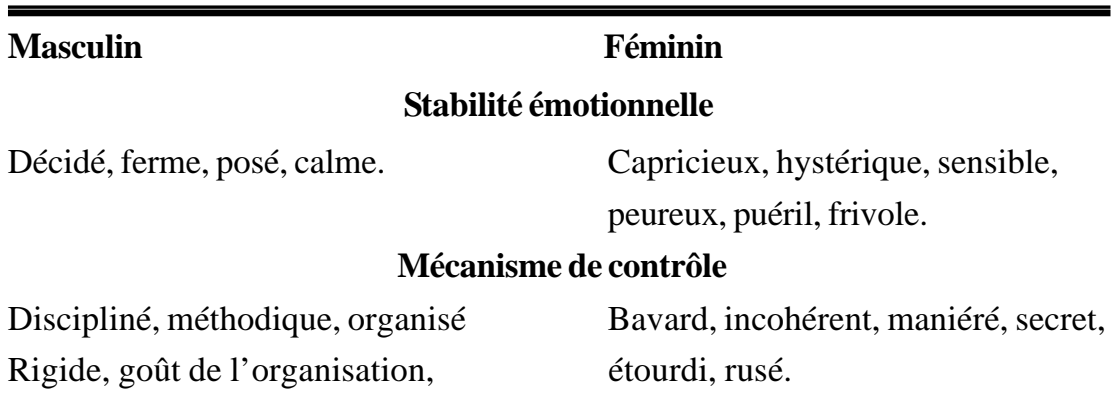

discret, franc.

\section{Autonomie, dépendance}

Patriote, goût du risque, indépendance. Besoin de se confier, besoin de plaire, coquet, soumis.

\section{Domination, affirmation de soi}

Besoin de puissance, besoin de célébrité

Faible.

Ambition, goût du commandement,

Domination, suffisant, sûr de soi,

Besoin de prestige, arriviste,

Besoin de s’affirmer.

\section{Agressivité}

Combatif, cynique, goût pour la lutte.

Rusé.

\section{Niveau d'activité}

Fougueux.

Passif.

\section{Acquisition}

Égoïste, matérialiste.

Curieux.

\section{Qualités intellectuelles, créativité}

Créateur, lucide, objectif, goût pour les

Intuitif.

idées théoriques. Aptitudes pour les

sciences, les mathématiques,

sceptique, raisonneur.

\section{Orientation affective, sexualité}

Obscène.

Caressant, compatissant, doux, pudique, goût pour la toilette, besoin d'avoir des enfants, besoin d'amour. 
Il nous semble important de prendre conscience des stéréotypes avec lesquels nous fonctionnons. Il apparaît ici que dans la différence des genres, il y ait un peu de biologique et beaucoup de culturel. En effet, la femme est éduquée pour séduire, plaire à l'autre ; l’homme, pour dominer, intervenir, agir, être lui-même. La personnalité féminine ou masculine se définit avec les valeurs et comportements du genre auquel ils appartiennent. On a attribué aux femmes des traits de personnalité qui ne sont pas innés mais acquis. Tous les être humains, dès la naissance, sont soumis à l'apprentissage d'une infinité de comportement en relation avec leur condition d'êtres culturels. En général, le garçon est élevé comme sujet autonome et la fille en fonction de l'autre, en accentuant sa dépendance, ainsi se structure son amour et son désir. On ne lui apprend pas à demander mais à pleurer, implorer, feindre ou se taire ; elle n'aura pas beaucoup d'assurance et sa préoccupation se réduira, fondamentalement, à vouloir plaire aux autres. En somme, la femme est faite pour être épouse.

Une brève traversée des théories relatives à l'institution sociale nous amènera à la conclusion que les femmes sont rendues invisibles sur deux plans : en tant qu'actrices sociales, voire même en tant qu'êtres humains ; et en tant que groupe socialement construit. Ce qui est corrélatif de leur sur-visibilisation comme êtres pensés plus naturels que les hommes. Cette construction de l'inégalité entre hommes et femmes reflète les différences de traitement qui existent dans la société. La puissance du chef de famille par exemple constitue un des points les plus problématiques de la théorie classique du droit naturel. L’idée d’une différence naturelle, voire d'une disparité des sexes interprétée plus ou moins explicitement dans le sens de la hiérarchie, y est soutenue ou considérée comme une évidence, même si des qualités spécifiques sont reconnues aux femmes. Qu’en est-il des théories économistes

\section{De l'économie de sexe au sexe de l'économie}

L’histoire des idées nous montre que les différents états traditionnels (noblesse, bourgeois, clergé, etc.) ne correspondent plus à la nouvelle vision que la société a d'elle-même. L'assomption de la richesse invite à penser avec de nouvelles catégories l'organisation sociale. En effet, pour les physiocrates, la nation est réduite à trois nouvelles classes de citoyens : la classe productive, la classe des propriétaires et la classe stérile. La classe productive est composée de cultivateurs, fermiers et ouvriers de la campagne. La classe des propriétaires comprend le souverain, les possesseurs de terres et ceux qui bénéficient de la dîme ${ }^{4}$. La classe stérile est formée des artisans, manufacturiers, marchands et plus généralement de tous les citoyens occupés à d'autres travaux que ceux de l'agriculture. 
On retrouve la même démarche chez Smith : ce sont aussi les catégories économiques qui définissent les classes sociales. Mais pour lui, l'agriculture n'est pas la seule source de richesse. On sait qu'il divise le produit annuel de la nation en trois parties : la rente de la terre, les profits des capitaux, les salaires du travail. Ce produit constitue ainsi un revenu à trois différentes classes : ceux qui vivent de rentes, ceux qui vivent de salaires, ceux qui vivent de profits. Ce sont donc les différents types de revenus qui définissent les classes sociales. Donner un fondement économique à la sociologie implique de penser la société comme mobile. Smith introduit ainsi par rapport aux physiocrates trois modifications qui sont lourdes de conséquences :

- Il rejette d'abord leur analyse des sources de la richesse ; pour lui la terre n'est qu'une source de richesse parmi d'autres.

- Il élabore ensuite une sorte de sociologie dialectique. Il y a séparation des classes sociales dans le moment de la production et unité des classes sociales dans le moment de la consommation.

- Smith reprend enfin la distinction productif/improductif des physiocrates. Mais il lui donne un sens nouveau. Il ne la situe plus à l’intérieur de la sphère de la richesse, mais en fait la ligne de séparation entre l’État et la société civile.

John Stuart Mill, célèbre économiste politique et féministe libéral du 19e siècle, figure dominante de la philosophie et de l'économie politique, soutient pour sa part que, la doctrine du libre échange a des fondements aussi solides que le principe de la liberté individuelle. D’où la nécessité de dissocier la légitimité de la liberté individuelle de celle de la liberté économique. Il pense donc l'accomplissement de l'individu dans une perspective de développement de la société civile. Condition préalable au développement social, le développement individuel dépend de la liberté de cultiver au plus haut degré les facultés de chaque individu. C'est dans ce libéralisme que prend racine l'analyse de l'assujettissement des femmes comme l'un des principaux obstacles qui s’opposent au progrès de l'humanité.

La représentation économique de la société implique un profond bouleversement sociologique. Un tel renversement des analyses traditionnelles, esquissé trop brièvement et trop schématiquement, est d'une ampleur telle que je ne saurais prétendre en venir à bout en si peu de pages. Les questions soulevées sont éminemment complexes. Y a-t-il une place pour la femme dans ce bouleversement ? Sans prétendre faire un traité de science économique, voyons comment ces théories ont fait l'économie du sexe dans la connaissance économique. 


\section{L'économie du sexe dans les théories économiques}

\section{L'objet de la connaissance économique}

- Les richesses matérielles

L'ouvrage d'Adam Smith, La Richesse des nations, suggère une première définition de l'objet de la connaissance économique. Pourquoi n'y aurait-il pas, en effet, une science des richesses, comme il y a une science de la lumière, des astres ou des végétaux ? La difficulté est de savoir ce qu'est une richesse. Des biens matériels d'abord, mais aussi des services nécessaires à l'existence, voire au bien-être (welfare). Déjà, on prend conscience qu'est richesse ce qui satisfait un besoin, cette fonction de satisfaction définissant l'utilité, constitue une notion clef du langage économique. Mais tandis qu'une longueur ou un poids ont une réalité objective, il n'y a pas d'utilité en soi, pas de valeur absolue. La notion de richesse est relative et subjective. Elle ne peut suffire à constituer une science. Les besoins pratiques d'une femme par exemple sont souvent liés aux conditions de vie difficiles, au manque de ressources.

- L'échange

Le concept de marché change de signification avec Adam Smith. Il n'est plus simplement un lieu particulier et localisé d'échanges : c'est la société tout entière qui constitue le marché. Il n'est pas seulement un mode l'allocation des ressources à travers une libre détermination des prix : c'est un mécanisme d'organisation sociale plus encore qu'un mécanisme de régulation économique. Chez Smith, le marché est un concept politique et sociologique, et c'est en tant que tel qu'il a une dimension économique. Il conçoit en effet les relations entre les hommes comme des relations entre des marchandises, dans la mesure où la nation est définie comme le système des besoins. Il pense l'économie comme fondement de la société et le marché comme opérateur de l'ordre social. Même s'il n’y a pas bienveillance réciproque entre les hommes, le lien social n'est pas rompu pour autant. Il continue de se maintenir pour des raisons « économiques ». A. Smith écrit à ce propos

La société peut alors subsister entre les hommes, comme elle subsiste entre des marchands, par le sentiment de son utilité, sans aucun lien d'affection : quoiqu'alors aucun homme ne tienne à un autre, par les devoirs ou par les nœuds de la gratitude, la société peut encore se soutenir, à l'aide de l'échange intéressé des services mutuels, auxquels on a assigné une valeur convenue. (Smith 1776 : 97).

Mais la valeur des biens et services n'existant pas en soi, elle ne se connaît qu'à travers l'échange. Ce qui est économique, c'est précisément ce qui est 
capable d'échange. L'acte économique se révèle comme tel seulement lorsqu'il y a passage, déplacement des biens entre les hommes. Il est social par essence. Et comme le transfert le plus élémentaire qui soit suppose deux sujets en présence, l'acte économique est un acte dichotomique. Il y a toujours la main qui reçoit et la main qui donne : une entrée et une sortie, un débit et un crédit. De la sorte, un acte gratuit ne rentre pas dans la sphère de la science économique, mais il reste à savoir si un acte aujourd'hui gratuit ne donnera pas lieu, dans un avenir plus ou moins éloigné, à une contrepartie.

En somme, les rapports entre les hommes sont compris comme des rapports entre des valeurs marchandes. Le lien économique qui relie les hommes comme producteurs de marchandises pour le marché, est considéré comme le véritable ciment de la société. La société existe, parce que chacun dit : « Donnez-moi ce dont j'ai besoin et vous aurez ce dont vous avez besoin vous-mêmes » (Smith 1776 I, 1 : 69). Le résultat d'un échange se traduit par un prix, c'est-à-dire un rapport des quantités transférées d'un sujet à un autre, que ce prix s'exprime en nature (prix relatif, le prix du riz en arachides par exemple) ou en monnaie (prix absolu, le prix du riz en francs).

Dans notre ordre social, les femmes sont « produites » utilisées, échangées par les hommes. Leur statut est celui des « marchandises ». Il obéit aux règles du marché, un marché qui a sa logique propre, liée à la logique d'un système d'exclusion et d'oppression des uns au bénéfice des autres. Comment cet objet d'usage et de transaction peut-il revendiquer un droit à la parole, et plus généralement, une participation aux échanges ? Quelle est la conception du corps féminin ? Nous pensons que cette situation d'oppression spécifique peut donner aux femmes la possibilité d'élaborer une « critique de l'économie politique », en tant qu'elles sont en position d'extériorité par rapport aux lois des échanges, tout en y étant incluses comme «marchandises ». On conçoit ainsi que la science des échanges se ramènera à une science des prix. Est économique tout ce qui peut se traduire par un prix. Mais pour savoir comment se forme le prix, il faut aller plus au fond du problème. Un troisième thème de recherche apparaît.

\section{- Les choix}

Une idée majeure préside à la naissance du problème économique : celle de limitation ou mieux encore d'inadaptation. L'homme porte en soi un besoin d'infini, et il bute constamment sur le fini de la création. Cette antithèse se traduit d'abord dans l'idée de rareté. Les besoins apparaissent comme innombrables, et les moyens pour les satisfaire sont limités. Il peut arriver aussi que les moyens soient suffisants, parfois même trop nombreux. Alors une autre notion intervient, celle d'inadaptation. Les biens ne sont pas forcément là où il en est besoin ni quand il en est besoin. Il faut les produire s'ils 
sont insuffisants, les réduire s’ils sont trop abondants. Il est nécessaire aussi d'en accélérer ou d'en retarder l'arrivée. L’acte économique apparaît alors comme l'acte d'adaptation par excellence. Si l'idée d'adaptation récapitule l’essence de l'économicité, si l’économie est en fin de compte la science de l'adaptation, la science économique est bien à même d'assurer à la fois la connaissance et la réalisation de cette adaptation, la connaissance et la réalisation ne cessant de se prêter un mutuel appui. De toute manière, avoir une attitude économique, c'est savoir choisir, et d'abord la fin à réaliser de préférence à une autre. Une fois celle-ci choisie, on doit décider du moyen le plus efficace parmi tous les moyens possibles. Ainsi, lorsqu'il est possible de mettre les fins en ordre de préférence, lorsque les moyens de parvenir aux fins sont limités et susceptibles d'application alternative, lorsque enfin la durée de réalisation est aussi limitée, le comportement prend la forme d'un choix qui est l'acte économique par excellence. Mais pour le profit de qui le principe « la fin justifie les moyens » est-il appliqué ?

À y voir de plus près, l'économie conventionnelle considère d'abord les normes et les priorités de chacun comme acquises, et ne se pose jamais la question de savoir pourquoi une personne veut ce qu'elle veut, et comment une personne rationnelle, qui s’occupe de son intérêt personnel, cherche ce qu'elle veut. Ensuite, elle se concentre sur les individus et sous-estime le rôle de l'action collective. Enfin elle se centre sur la valeur de la production du marché et du travail productif. À ce niveau, la distinction travail productif/ travail non productif nous aide à voir la place de la femme dans ce bouleversement de la représentation traditionnelle des hiérarchies et des utilités sociales.

Avec A. Smith,

le travail de quelques-unes des classes les plus respectables de la société, de même que celui des domestiques, ne produit aucune valeur. Le souverain par exemple, ainsi que tous les autres magistrats civils et militaires qui servent sous lui, toute l'armée, toute la flotte, sont autant de travailleurs non productifs. Il sont serviteurs de l'État, et ils sont entretenus avec une partie du produit annuel de l'industrie d'autrui. Leur service, tout honorable, tout utile, tout nécessaire qu'il est, ne produit rien avec quoi on puisse ensuite se procurer une pareille quantité de service... Quelques unes des professions les plus frivoles, doivent être rangées dans cette même classe : les ecclésiastiques, les gens de loi, les médecins et les gens de lettres de toute espèce, ainsi que les comédiens, les farceurs, les musiciens, les chanteurs, les danseurs d'opéra, etc, Smith 1776 : 414).

On peut en déduire que les fonctionnaires, les militaires, les prêtres et les juges sont considérés économiquement comme les parasites des véritables producteurs. La femme pour sa part, est une fois de plus occultée. Il faudra 
attendre John Stuart Mill connu pour avoir été non seulement le grand philosophe du libéralisme britannique, mais aussi le seul philosophe majeur du libéralisme à avoir fait de l'assujettissement des femmes une partie constitutive de son œuvre philosophique.

\section{"L'assujettissement" : véritable manifeste des droits des femmes}

John Stuart Mill (1992) a été très tôt convaincu du bien-fondé de l'égalité des sexes. C'est dans ce cadre qu'il publie en 1869 De l'assujettissement des femmes (Mill 1992) ${ }^{5}$ : un véritable manifeste des droits des femmes. L'assujettissement des femmes n'est donc pas seulement le résultat de leur exclusion de la vie publique ; il s'enracine dans la famille et les rapports de pouvoir qui s'y exercent, portant atteinte au plus profond de l'individualité des femmes : en les privant de toute autonomie financière, puisque tout ce qui est à elles est à leurs maris.

Mill affirme, certes, que les femmes doivent être libres de choisir la carrière qui leur convient sans être obligées de se marier pour subvenir à leurs besoins. L'accès égal à l'instruction et au travail rémunéré devait assurer leur autonomie financière, transformer le mariage d'obligation en choix, ce qui par ailleurs pouvait contribuer à résoudre la question de la surpopulation. Mais si elles choisissaient le mariage, les femmes devaient se plier à la division du travail qui « selon l'usage ordinaire charge l'homme de gagner le revenu et la femme de diriger les dépenses domestiques »(Mill 1992 : 94-98).

À ce niveau, il nous semble que la non prise en considération de " genre », et donc des rapports de pouvoir entre les genres, mérite d'être soulignée parce qu'elle met particulièrement bien en valeur le caractère rudimentaire de l'approche économique orthodoxe. Cette perspective nécessite un changement d'optique. Il ne s'agira pas « d'ajouter les femmes et remuer », c'està-dire simplement d'étendre le champ d'analyse aux femmes, sans pour autant s'interroger sur la dimension sexuée de la définition traditionnelle des concepts tels que le bien-être, les échanges et les choix économiques. En se situant, cette fois, non sur le plan de la contestation (préalable à toute construction), mais sur celui de l'orientation positive, le genre ne fournit-il pas ce ferment révolutionnaire capable de créer une civilisation solidaire qui ne soit pas une termitière, mais une communauté librement consentie de personnes responsables ? C'est l'objectif même de l'économie féministe.

\section{Le sexe de l'économie}

À la question qu'est-ce que les femmes ont apporté ou pourraient apporter à l'économie, il est intéressant de retourner la question et de se demander ce que la pratique de l'économie apporte aux femmes, ce qu'elles y trouvent pour elles-mêmes. Autrement dit, y a-t-il un apport spécifique des femmes à 
l'économie ? Mieux encore, si les femmes avaient le pouvoir, l'éthique et la politique économiques - le choix des priorités par exemple - seraient-elles les mêmes ?

\section{Vers le système économie/genre}

- Les courants de pensée dans les mouvements de femmes

La situation d'inégalité et d'injustice sociale détermine, à travers le monde, les grandes orientations de revendication des mouvements féministes. Il y a eu différentes tentatives d'organisation des femmes, seules ou appuyées par des hommes, pour revendiquer le principe d’égalité et de justice pour tous. Même volonté de mettre les femmes en position de sujets et non pas d'objets de discours ; mêmes demandes sociales et politiques d'égalité ; C’est sur la toile de fond de ces analogies qu'on peut remarquer les différences avec les étapes antérieures. Différence dans l'analyse économique : d’une part, le critère de l'indépendance financière joue pour caractériser non seulement l'autonomie sociale de la «travailleuse », mais aussi l'autonomie familiale d'un individu salarié ; d'autre part, le statut même du travail des femmes est pensé dans une globalité où le travail domestique n’est pas séparé du travail social, où la fonction de reproduction n'est pas dissociée du système de production.

Le féminisme peut donc être défini comme le mouvement d'idées et d'actions ayant une volonté de produire une action et un savoir renouvelé susceptible de contribuer à la fois à l'élimination de la perspective androcentrique toujours dominante dans les sciences de l'homme, et à la redéfinition de nouveaux rapports hommes/femmes. À y voir de plus près, on peut identifier trois grands courants qui ont marqué le mouvement de femmes : le féminisme radical, le féminisme égalitaire ou réformiste, le discours féministemarxiste.

Pour le féminisme radical, la première division de classes se serait établie à partir de la différence biologique liée à la reproduction. Il ne conçoit pas que la nature puisse servir d'explication à l'infériorisation des femmes et soutient que celle-ci procède plutôt des rapports d'oppression du système patriarcal. Il se donne par conséquent la mission d'abolir ces rapports. Il se propose en somme de redéfinir les rapports sociaux en dehors de toute contrainte biologique.

Le féminisme égalitaire ou réformiste situe l’inégalité dans les modèles de socialisation en fonction du sexe qui confinent les femmes à des situations d'infériorité. Il revendique une situation plus égalitaire entre les hommes et les femmes à travers des réformes pour améliorer les conditions sociales, 
économiques et juridiques des femmes. Tout en excluant de sa stratégie la rupture avec l'ordre établi.

Sur quoi est centré le discours féministe-marxiste ? Dans l'économie politique de Karl Marx, l'analyse de la production marchande et du profit retiré du travail salarié éclipsait les profits que pouvaient retirer le capital et la famille de la production domestique non marchande des femmes. C'est pourquoi Karl Marx et Engels ne voient l'émancipation des femmes que comme une conséquence de celle du prolétariat. Ainsi associant la critique morale à l'analyse scientifique de l'injustice, de l'exploitation et de la domination de classe dans la société capitaliste/industrielle, ils lancent un appel universel à l'action (au-delà des frontières culturelles, nationales ou religieuses) pour l'abolition de cette domination.

L'intérêt de l'œuvre de Marx et d'Engels réside moins dans leur analyse de la position des femmes dans la société moderne que dans les armes conceptuelles et politiques qu'elle fournira aux analyses féministes de la société. Un grand nombre de courants féministes, ont entretenu avec leur pensée des rapports à la fois étroits et très critiques. Si en règle générale, les féministes « socialistes » ont longtemps subordonné la libération des femmes à l'émancipation du prolétariat, l'accent porté par les mouvements de libération des femmes sur l'auto-organisation et l'auto-émancipation fut souvent inspiré de cette philosophie de la praxis, dans laquelle se rencontrent changement des circonstances et transformation des consciences. Tandis que l'analyse marxiste de l'oppression des femmes fut l'objet d'une critique systématique, la méthode marxiste a souvent fourni les armes théoriques et politiques d'une telle critique. Parce que dans le concept de classe, les groupes dominant et dominé n'existent pas en tant que tels indépendamment du rapport de domination qui les produits, ce cadre théorique a permis, comme l'a remarqué Christine Delphy, de penser les hommes et les femmes comme deux groupes antagoniques qui sont socialement construits de manière hiérarchique et interdépendante(Delphy 1998 : 28).

Le discours féministe-marxiste est donc centré sur l'exploitation et l'oppression. C'est l'absence des femmes dans les rapports de production et leur dépendance économique qui est la cause de leur exploitation ; à cela, il faut ajouter le patriarcat, c'est-à-dire la suprématie des hommes sur les femmes en tant que groupe. Les approches féministes-marxistes reconnaissent que les deux niveaux d'oppression se superposent et s'articulent. Sur le plan économique, l'approche genre ${ }^{1}$ met en évidence l'interaction complexe des aspects social, économique, politique et idéologique, au lieu de voir dans les éléments économiques un facteur déterminant dans tous les cas. D’où son intérêt. 
- L'approche genre, un outil d'analyse scientifique

On peut parler de science quant il y a un objet clairement identifié, des méthodes et des outils. L'économie, considérée comme une science « exacte » se veut quantitative, non normative, objective et dotée d'un statut supérieur. Il est vrai qu'elle se montre à la hauteur de l'image ambitieuse, moderniste et masculine qu'elle a d'elle-même. Tout débat télévisé sérieux par exemple comprend un économiste, tout comité consultatif auprès du gouvernement, quel que soit le sujet, comprendra un économiste, et tout journal ou magazine généraliste comporte, par numéro, au moins une ou deux interviews avec un économiste. Bref, le facteur le plus frappant dans la présentation de l'économie comme une science " exacte » et masculine, hostile à tout regard féminin réside dans son caractère de forteresse protégeant le paradigme néoclassique ${ }^{6}$. La théorie économique, dans sa forme dominante, est synonyme de l’idéologie libérale du marché.

L'approche genre pour sa part, délimite un objet portant sur les relations entre hommes et femmes, un cadre théorique qui repose sur la théorie des rôles et des besoins, et dégage des instruments de mesure. Ainsi, le concept genre apporte une importante contribution épistémologique à l'avancement des connaissances scientifiques ; l'apport de l'analyse genre se situe principalement à deux niveaux :

- Au niveau de la démonstration du caractère construit, par opposition au caractère naturel des catégories homme/femme

- Au niveau de la prise en compte des rapports politiques et conflictuels, c'est-à-dire du pouvoir.

La portée scientifique de l'outil d'analyse genre est surtout sa pertinence pour toute forme de situation d'inégalité qu'elle soit liée au sexe, à l'ethnie ou à l'âge. L'analyse genre permet de produire un savoir renouvelé et intégré, susceptible de contribuer à la fois à l'élimination de la perspective androcentique toujours dominante dans les sciences de l'homme et à la redéfinition de nouveaux rapports hommes/femmes.

\section{Le genre: un concept et une approche nouvelle de l'économie}

Le concept genre se fonde sur le fait que les différences, les rôles, les positions entre les hommes et les femmes découlent d'une construction sociale et culturelle, basée sur une inégalité fondamentale. Par conséquent, une approche de socialisation basée sur l'équilibre des relations entre les hommes et les femmes devrait aboutir à la construction d'une société plus juste et plus équitable. L'approche genre repose sur des principes d'équité, d'égalité et de justice sociale. Dans le domaine économique, elle a permis d'arracher les femmes à leur isolement et aux rapports toujours médiatisés par les hom- 
mes qu'elles entretenaient. Si aujourd'hui les grandes luttes du féminisme sont devenues légitimes, il n’est pas certain que la « libération de la femme » représente un mouvement achevé (heureusement d'ailleurs puisqu’on achève un animal lorsqu'il n'est plus bon à rien). Les féministes cherchent à élaborer une conception de la société, de la citoyenneté, de la politique au sens de participation à la vie de la cité, qui ne soit plus basée sur la prédominance de qui que ce soit (les hommes en l'occurrence). En d'autres termes, il s’agit de repenser l'humain, le rapport à l'autre.

À ce niveau, le sentiment est de plus en plus partagé que les problèmes de la promotion effective des femmes appellent des solutions qui engagent aujourd'hui la responsabilité et le devenir de l'humanité dans son ensemble. Il a été reconnu tant dans les pays du nord que dans ceux du sud qu'il ne peut y avoir développement durable ${ }^{7}$ sans implication des femmes ou sans prise en compte du rôle, de la place et de la contribution des femmes dans le processus de création de la valeur ajoutée et de la richesse. Une approche du concept de genre nous situe dans une position d'analyse réaliste non seulement des processus personnels, mais des processus sociaux et économiques globaux. C'est dans se sens que nous pouvons voir l'émergence de l'économie féministe.

Plusieurs théories sont à la base des mouvements d'action ou de réflexion sur les femmes. Sur le terrain du développement par exemple, les différents cadres conceptuels vont se traduire aussi dans les programmes pour les femmes. Deux grands courants de pensée retiennent notre attention. Il s’agit de l'IFD (Intégration des femmes au développement) influencée par les approches libérales et le GED (Genre et Développement) caractérisé par les approches socio-marxistes.

\section{Le genre dans les stratégies d'auto-insertion des femmes}

- L'intégration des femmes au développement et la pensée libérale L'intégration des femmes au développement est apparue au début des années 1970, en remplacement des programmes d'assistance des années 1950-1970 dont les résultats n'avaient pas été satisfaisants. Elle avait pour objectif d'éliminer les discriminations et d'améliorer la condition féminine, c'est-à-dire, les besoins pratiques. Cette approche s'inspire de la pensée libérale et s'inscrit dans la perspective de la théorie traditionnelle de la modernisation. Elle pense que les inégalités entre les sexes s'estomperont d'elles-mêmes, lorsque les femmes deviendront des partenaires économiques à part entière. Elle est utilisée dans le cadre des politiques de croissance économique et d'efficacité, avec pour objectif d'amener les femmes pauvres à augmenter leur productivité, par des projets générateurs de revenu et de faire en sorte que le développement soit plus efficace et rentable grâce à la contribution économique des femmes. 
Or, les besoins pratiques des femmes ont tendance à être immédiats, à court terme, ils sont particuliers à certaines femmes, il sont liés aux besoins quotidiens : nourriture, logement, revenus, santé des enfants, etc, ils sont facilement identifiables par les femmes et peuvent être satisfaits par des éléments précis. A cause de leur position sociale défavorable, l'amélioration des femmes passe par un changement de leurs conditions sociales. La satisfaction des besoins pratiques peut améliorer les conditions de vie des femmes, mais en général n’altère pas les rôles et les rapports traditionnels.

Le bien-être qui se dégage ici ne remet pas en cause la subordination des femmes. Il cherche à aider les groupes les plus vulnérables, où sont placées les femmes, en donnant la priorité à la production. Il crée chez elles une dépendance dommageable. Ainsi, tout en relativisant les déterminations biologiques des inégalités entre les sexes, la logique de l'IFD repose sur un certain déterminisme social. Elle revendique une situation plus égalitaire entre hommes et femmes, au travers de réformes pour améliorer les conditions sociales économiques et juridiques des femmes ; toutefois, elle exclut de sa stratégie une rupture avec l'ordre établi. De manière générale, l'approche IFD a perçu les femmes comme des bénéficiaires et non comme des actrices du développement, renforçant de ce fait leur passivité et leur dépendance. Une telle approche n'est pas porteuse d'alternative, elle ne critique pas les structures d'oppression productrices d'idéologies sexistes, de lois et pratiques inégalitaires. D’où le changement de perspective à travers le GED.

- L’approche genre et développement et la pensée marxiste

Dans les années 80, il y eut une remise en cause des projets IFD qui, tout en améliorant les conditions sociales des femmes, ne changent pas, quant au fond, leurs positions sociales. C'est dans ce contexte que la perspective genre va émerger comme une alternative. Elle a une vision plus globale. Les fondements théoriques s'inscrivent dans le féminisme marxiste, mettant l'accent sur le secteur de la production aux dépens des aspects reproductifs du travail et de la vie des femmes. Cette approche établit un lien entre les relations de production et de reproduction et prend en compte tous les aspects de la vie de la femme. A la différence des autres cadres théoriques, son objet n’est pas la femme en tant que telle, mais les réalités sociales qui façonnent les perspectives et attribuent les responsabilités et des attentes spécifiques aux femmes et aux hommes. Elle introduit la perspective de l'analyse sexospécifique qui permet de prendre en compte les différences et les contraintes qui affectent et les hommes et les femmes dans les rapports qu'ils entretiennent dans la production. Ici, l'analyse genre n'a pas pour objet la femme en tant que telle, mais les réalités sociales qui façonnent les perceptions et attribuent des responsabilités et des attentes spécifiques aux femmes et aux hommes. 
Remarquons toutefois qu'en faisant valoir que l'oppression des classes prime sur toute autre forme d'oppression, l'approche GED pèche par réductionnisme. Il semble subordonner la libération de la femme à la suppression de la lutte des classes. Un tableau comparatif ${ }^{8}$, permet de rendre compte des différences d'approche.

\section{Tableau 2 : les différentes approches}

\section{Intégration des femmes au Genre et développement (ged) développement (ifd)}

\section{L'approche}

Une approche qui considère les Une approche du développement femmes comme étant le problème

\section{Le centre d'intérêt}

Les femmes

Les rapports femmes-hommes

\section{Le problème}

L'exclusion des femmes (qui Les relations de pouvoir inégales (riches et représentent la moitié des respauvres, femmes et hommes) qui empêchent sources potentielles de production) du processus de développement un développement équitable ainsi que la pleine participation des femmes.

\section{L'objectif}

Un développement plus efficient, plus efficace.

Un développement équitable et durable, où les femmes et les hommes prennent les décisions.

\section{La solution}

Intégrer les femmes au processus de développement existant.

Accroître le pouvoir des plus démunis et des femmes.Transformer les relations non égalitaires.

\section{Les stratégies}

Projets pour les femmes

Volets femmes

Projets intégrés Accroître la Identifier/considérer les besoins pratiques et déterminés par les femmes et les hommes en vue d'améliorer leur conditionTraiter en productivité des femmes Accroître la capacité des femmes à effectuer les tâches traditionnellement liées à leur rôle même temps des intérêts stratégiques des femmesFaire face aux intérêts stratégiques des pauvres par un développement axé sur le genre. 
Aucune de ces deux expériences ne permet à mon sens de fonder une stratégie alternative permettant d'ouvrir la voie à une économie éthiquement durable. Les réformes économiques, qu'elles se fassent au niveau micro, méso ou macroéconomique, sont marquées de préjugés masculins qui perpétuent le désavantage relatif des femmes. L'économie féministe rétablit la visibilité des liens toujours existants entre genre et économie. Elle se penche plus sur un thème spécifique, à savoir le rôle des femmes. Elle démontre que l'interdépendance entre genre et économie découle du triangle :

- F relations humaines,

- F façons de penser le genre

- F et réalités économiques.

Or dans la vie économique quotidienne, ce triangle est occulté, et pourtant, les femmes en vivent la réalité tous les jours. A partir de la question du genre, le quotidien apparaît par exemple comme élément important. Le quotidien ici c'est la vie de tous les jours. Le quotidien, c'est le combat pour vivre, survivre aujourd'hui, pour chercher du travail, pour faire la cuisine, pour laver les enfants et le linge, pour échanger des gestes d'amour, pour trouver un sens immédiat à l'existence. Le quotidien, c'est le monde domestique, le monde des relations courtes, des relations plus directes, qui sont parfois capables de changer les relations plus larges. Le quotidien des femmes s'introduit dans la science dite universelle pour lui rappeler le concret, les choses qui sont nécessaires à la vie ou à la survie. Le quotidien c'est la routine, les habitudes de chaque jour. Le quotidien, ce sont nos histoires personnelles, nos sentiments face aux événements, nos réactions concernant différentes questions de l'actualité.

\section{Conclusion}

Les recherches sur les femmes ont mis en évidence l'influence des concepts et des définitions utilisés sur la perception des rapports sociaux. L'histoire de l'humanité semble tourner autour des questions de liberté humaine et du contrôle de la production et de la reproduction. Ce qui s'est traduit par des luttes de domination entre nations, races, et classes sociales ; mais quelles que soient la société, la race ou la nation, la domination de la femme par l'homme est restée une constante. Les hommes ont utilisé leur pouvoir sous forme de violence et de contrat social pour contrôler le travail d'autres hommes. Ce qui les a conduits à l'utilisation du pouvoir afin d'imposer des limites aux femmes. Même si la différence des sexes est naturelle, la situation faite aux femmes ne l'est pas. Celle-ci est culturelle, idéologique. Les philosophes universalistes ont introduit «tranquillement » une brèche dans la cohérence 
de leur système en excluant les femmes, ces êtres humains, de leur propre définition de l'humanité.

La réflexion sur la relation entre homme et femme a connu des moments importants dont chacun correspond à la prééminence d'un modèle théorique particulier. Partis d'un modèle « unidimensionnel » enraciné dans la dimension biologique, les chercheurs ont élaboré des modèles «bi » ou « pluridimensionnels » pour rendre compte de la complexité croissante de la catégorie du genre et des ses composantes. La réappropriation de l'identité féminine passe alors par une sorte d'injonction à l'altérité pour parer aux dangers d'une indifférenciation des sexes ou de leur neutralisation sous le signe du masculin. Rousseau leur paraît permettre de penser à la fois la féminité et l'égalité. Mais si nul mieux que lui n’a si fermement posé les conditions de l'égalité, on ne doit pas oublier qu'il en excluait les femmes. Nul n'aura mieux fait de l'altérité sexuelle la condition même de l'amour, nul n'aura plus insisté sur les missions de l'éducation maternelle. Nul mieux que lui n'aura vanté la féminité, mais il l'enfermait dans la douce royauté de la femme en son foyer. À la recherche de ce qui « est femme », ne risque-t-on pas, dans le même flou arbitraire que Rousseau, de perdre le fameux « reste » ?

S'il fallait indiquer schématiquement le parcours poursuivi, on pourrait dire, qu'à la métaphysique des sexes, fondant l'infériorité des femmes, se substitue progressivement l'apologie du féminin qui concerne dès lors l'un et l'autre sexe. Ce déplacement se repère dans des domaines très divers, que ce soit en philosophie, en théorie sociale et politique, en logique, ou dans le féminisme. Il se développe en effet un peu partout une mise en perspective critique de la totalité, du clos, du logocentrisme, de la maîtrise, au profit du " pas tout », de l'infini, de l'ouvert, du décentrement, de l'illimité. Certes, l'ordre du tout est incontournable -ordre phallique, ordre métaphysique mais il est « pas tout ». On assiste ainsi à une perte de crédit de la « modernité » comme maîtrise du Sujet, qui pourra être assimilée à une perte de crédit de la " virilité ». C'est à partir de l'individu et de sa nature qu'il faudra désormais penser et résoudre le problème de l'institution du social. C'est dans ce bouleversement que se posent les conditions de possibilité d'une meilleure économie.

Aujourd'hui, l'étoile filante des grandes institutions financières internationales tourne autour de l'économique, de la recherche permanente du profit. On assiste à un déséquilibre entre le social et l'économique. Le modèle actuel est tourné vers la promotion des outils et des moyens, et non vers celle des personnes. Ne risquons-nous pas de perdre ce qui nous reste de plus précieux, à savoir notre identité propre et nos valeurs socioculturelles si diversifiées? 
Dans l'ordre politique, l'autonomie, est en train d'émerger. C'est en son nom que se mènent aujourd'hui des combats décisifs dans le cadre de vie et dans la culture. Elle a également une dimension économique qu’il s’agit aujourd'hui d'approfondir. Le concept d'autonomie est en effet un instrument décisif de critique de l’idéologie économique pour laquelle l’égal suppose le commensurable, l’humain.

\section{Notes}

1. Les théories relatives au Genre se sont développées en cette fin du XXe siècle. On les trouve dans les nombreux ouvrages publiés par les féministes francophones, mais surtout par les féministes anglo-saxonnes, spécialement par les Nord-Américaines. Nous citerons ici les travaux de la philosophe belge Françoise Collin (1992). Voir aussi Julia Kristeva (1983) et Luce Irigaray (1977).

2. On parlera d'approche genre pour définir la démarche scientifique qui prend en compte la relation sociale de sexe dans l'analyse et la transformation de la réalité sociale.

3. Entendu au sens de décomposition, pourriture.

4 On sait que pour les physiocrates, le souverain est considéré comme le propriétaire de toutes les terres du royaume. C'est ce qui justifie le fait qu'il puisse lever l'impôt.

5. Avec ses Principes d'économie politique (1848), Stuart Mill renoue avec la tradition des pères fondateurs du libéralisme économique. Moraliste et économiste, il pose le problème des rapports entre la question économique et les questions sociales : l'ordre social ne trouve son ultime justification que dans la poursuite du bonheur pour tous. C'est à lui que l'on doit la distinction entre les « lois de production », de caractère physique ou naturel, et en tant que telles parfaitement universelles, et les « lois de répartition », de caractère social, et susceptibles à ce titre d'être subordonnées à des objectifs moraux ou, si l'on préfère, politiques.

6. Les néoclassiques reprennent les idées des classiques : l'économie de marché, la libre concurrence, pas ou peu d'intervention de l'État, le libéralisme économique, la neutralité de la monnaie, etc. De plus, l’équilibre est général, c'est-à-dire simultané sur tous les marchés. Leur raisonnement se fait au niveau microéconomique à partir du comportement des agents qui se rencontrent sur les marchés. Les agents sont supposés être rationnels et chercher à maximiser leur utilité ou leur profit. Les marchés sont supposés de concurrence pure et parfaite. En somme, la théorie néoclassique est une théorie des prix relatifs et de l'allocation de ressources considérées, par définition, comme rares.

7. Le développement durable apparaît comme une nouvelle forme de développement de l'humanité respectant l'environnement global et les équilibres écologiques fondamentaux qui régissent tant les milieux continentaux qu’océaniques. L’objectif final est de répondre aux besoins actuels sans compromettre l'aptitude des générations futures à répondre aux leurs. En 
résumé, c’est un développement qui répond aux besoins du présent sans compromettre la capacité des générations futures de répondre aux leurs.

8. Tiré de : Un autre genre de développement. Un guide pratique sur les rapports femmes-hommes dans le développement. CCCI, CI Match, AQOCI Ottawa, 1991.

\section{Références}

Alternatives Sud, 2001, « Et si l’Afrique refusait le marché ? », cahiers trimestriels Vol. VIII, 3.

Aristote, 1963, De la génération des animaux, livre IV, Paris : Les Belles Lettres. Aristote, 1968, La Politique I, 1260a, Paris : Les Belles Lettres.

Aristote, 1982, De Anima II, 1, 412a, Paris : Les Belles Lettres.

Badinter, Élisabeth, 1986, L'Un est l'Autre : des relations entre hommes et femmes Paris : Odile Jacob (coll. Points, 29).

Bassiliat, Jeanne, 1992, Relations de Genre et développement, Paris : ORSTOM.

Bassiliat, Jeanne, Verschuur, Christine, 2000, Le genre : un outil nécessaire, Cahiers Genre et Développement, $n^{\circ} 1$.

Bassiliat, Jeanne, Verschuur, Christine, 2002, Genre et économie : un premier éclairage, Cahiers Genre et Développement, $\mathrm{n}^{\circ} 2$.

CDEACF et Relais Femmes, 1997, Qu'est-ce que le Féminisme ? Université de Montréal : Collection « Les sciences sociales contemporaines ».

CETRI, 1999, Rapport de genre et mondialisation des marchés, Cahiers trimestriels, Alternatives Sud, volume V (4), l'Harmattan.

Collin, Françoise, 1984, D’amour et de raison. Les cahiers du GRIF, Paris, Cité d'Angoulême.

Collin, Françoise, 1992, « Différence et différend », in Duby, G., Perrot, M. (dir.), Histoire des femmes, t. 5, Paris : Plon.

Collin, Françoise, 1992, « Le sexe des sciences. Les femmes en plus », in Françoise Collin, dir., Parmi les femmes et les sciences. Paris : Éditions Autrement, Série Sciences en société, $\mathrm{n}^{\circ} 6$.

Collin, Françoise, 1992, « Praxis de la Différence : notes sur le tragique du sujet », Cahiers du GRIF, n 46, Printemps, Paris, Cité d'Angoulême 75001.

Collin, Françoise, 1999, L'homme est-il superflu, Hannah Arendt, Paris : Odile Jacob.

Collin, Françoise, Pissier, Evelyne et Varikas, Eleni, 2000, Les femmes, de Platon à Derrida. Une anthologie critique, Paris : Plon, 830 p.

Delphy, Christine, 1998, L'ennemi principal. Economie politique du patriarcat, Paris : Édit. Syllepse, coll. Nouvelles questions féministes.

Delphy, Christine, 2001, L'ennemi principal 2. Penser le genre, Paris : Édit. Syllepse, coll. Nouvelles questions féministes.

« Femme », 1968, Encyclopaedia Universalis, vol. 6, Paris.

Gebara, Yvonne, 1999, Le mal au féminin. Réflexions théologiques à partir du féminisme, Paris : l'Harmattan, 247 p

Héritier, Françoise, 1978, Le Fait féminin, Paris : Fayard. 
Héritier, Françoise, 1996, Masculin/Féminin : La pensée de la différence, Paris : Éd. Odile Jacob.

Hobbes, Thomas, 1996, Du citoyen, Paris : Le livre de Poche.

Hobbes, Thomas, 1999, Léviatan , trad. F. Tricaud, Paris : Dalloz.

Irigaray, Luce, 1974, Speculum de l'autre femme, Paris : Éd. de Minuit.

Irigaray, Luce, 1977, Ce sexe qui n'en est pas un, Paris : Éd. de Minuit : . 217 p.

Irigaray, Luce, 1989, Le Temps de la Différence. Pour une révolution pacifique, Paris : Le Livre de poche, Biblio Essais.

Jacquet, Isabelle, 1995, Développement au masculin/féminin : le genre, outil d'un nouveau concept, Paris : l'Harmattan, 184 p.

Kristeva, Julia, 1983, Histoire d'amour, Paris : Denöel.

Mathieu, Nicole, 1991, Identité sexuelle/sexuée/de sexe : trois modes de conceptualisation du rapport entre sexe et genre dans l'anatomie politique. Catégorisations et idéologie du sexe, Paris : Côté-Femmes.

Mathieu, Nicole, 1992, "Études féministes et anthropologiques », Dictionnaire de l'Ethnologie et de l'Anthropologie, Paris : PUF.

Mill, John Stuart, 1848, Principes d'économie politique, Collection « Les auteur(e)s classiques ».

Mill, John Stuart, 1992, De l'Assujettissement des femmes, Paris : Avatar.

Platon, 1961 « Le Timée », Euvres complètes, $3^{\circ}$ éd. t. II, Paris : Gallimard.

Platon, 1984, « La République, V, 452-456 », Euvres complètes, t. I, Paris : Gallimard, La Pléiade.

Platon, 1984, « La République, VII, 452e », Euvres complètes, t. I, Paris : Gallimard, La Pléiade.

Proudhon, Pierre Joseph, 1990, De la justice dans la Révolution et dans l'Église, Paris : Fayard, vol. VI.

Rosanvallon, Pierre, 1979, Le capitalisme utopique critique de l'idéologie économique, Paris : Éditions du Seuil.

Rocheblave-Spenle, Anne-Marie, 1964, Les Rôles masculins et féminins, Paris : PUF, ouvrage publié avec le concours du CNRS, cité par E. Badinter, p. 164.

Rousseau, Jean-Jacques, 1966, Émilie ou de l'Éducation, Paris : Flammarion.

Smith, Adam, 1776, Recherches sur la nature et les causes de la richesse des nations, Collection « Les auteur(e)s classiques ».

Un autre genre de développement. Un guide pratique sur les rapports femmeshommes dans le développement, 1991, CCCI, CI Match, AQOCI Ottawa. 